\title{
A New Thiopurine S-Methyltransferase Haplotype Associated With Intolerance to Azathioprine
}

\author{
Lara Colleoni, BSc', Dimos Kapetis, MSc', Lorenzo Maggi, MD', \\ Giorgia Camera, MD', Eleonora Canioni, BSc', Paola Cavalcante, PhD', \\ Nicole Kerlero de Rosbo, PhD', Fulvio Baggi, PhD', Carlo Antozzi, MD', \\ Paolo Confalonieri, MD', Renato Mantegazza, MD', and Pia Bernasconi, PhD'
}

\begin{abstract}
The authors have analyzed single nucleotide polymorphisms in the thiopurine S-methyltransferase (TPMT) gene in the context of efficacy and toxicity of azathioprine (AZA) to determine possible genotype-phenotype correlations between TPMT allelic variants and response to AZA treatment in 76 Italian patients with myasthenia gravis. They confirm known intronic and exonic TPMT polymorphisms that do not correlate with AZA responses and demonstrate a novel intronic polymorphism in a patient intolerant to AZA. Most importantly, they show that of the 22 AZA-intolerant patients, all 5 who carried mutations of the intolerance-linked haplotype TPMT*3A also carried the intronic TI40+II4A (rs3931660), all 3 mutations being part of a new haplotype designated TMPT*3E. TPMT*3E was not observed in unresponsive or responsive patients. The association of TPMT*3E with AZA intolerance and its frequency must be ascertained in larger, ethnically different cohorts. Nevertheless, in view of the highly significant association $(P s i m=0.0026)$ between TPMT*3E and AZA intolerance in the study, this new haplotype should be taken into consideration in pharmacogenetic profiling for AZA.
\end{abstract}

\section{Keywords}

thiopurine S-methyltransferase, azathioprine, single nucleotide polymorphism, haplotype, myasthenia gravis

Azathioprine (AZA), a purine antagonist that inhibits the cell cycle, is usually used as an immunosuppressant, steroid-sparing drug ${ }^{1}$ in autoimmune diseases, such as myasthenia gravis $(\mathrm{MG}){ }^{2}$ multiple sclerosis, ${ }^{3}$ and rheumatoid arthritis. ${ }^{4}$ To be active, AZA requires bioconversion to 6-mercaptopurine (6-MP) by glutathione S-transferase (GST). ${ }^{5}$ 6-MP can be metabolized either through the hypoxanthine phosphoribosyl transferase (HPRT) pathway to active 6-thioguanine nucleotides (6-TGN) or through the thiopurine S-methyltransferase (TPMT) pathway to inactive methyl-thiopurine metabolites. ${ }^{6}$ Active 6-TGNs incorporate into DNA, causing breaks in DNA strands, and thereby interfere with RNA and protein synthesis. Response to AZA is determined by which pathway is being favored for 6-MP metabolism. While balanced use of both HPRT and TPMT pathways results in responsiveness to AZA, hyperactivity of TPMT skews the balance toward the TPMT pathway and results in unresponsiveness to AZA with no pharmacological effects ${ }^{7}$; in contrast, low TPMT activity skews the balance toward the HPRT pathway, resulting in increasing side effects due to accumulation of $6-\mathrm{TGN}^{8}$ (Supplementary Figure S1). While moderate side effects are reversible upon appropriate reduction in AZA dosage, AZA treatment must be withdrawn when these include hematological or liver toxicity. ${ }^{9,10}$
Pharmacogenetic analyses indicate that therapeutic efficacy and/or toxicity of AZA could be determined, to some extent at least, by polymorphisms in the TPMT gene. The TPMT gene, located on chromosome 6 (6p22), consists of 9 introns and 10 exons and encodes a 245-amino-acid enzyme, ${ }^{11}$ with no known endogenous substrate or clear biological role. An early study showed a trimodal distribution of TPMT activity associated with a particular haplotype, thereafter referred to as $T P M T * 3 \mathrm{~A}$ : $88.6 \%$ individuals were wild-type with normal enzyme activity, $11.1 \%$ had intermediate TPMT activity and were heterozygous for $T P M T^{*} 3 \mathrm{~A}$, and the remaining $0.3 \%$, with very low TPMT activity, were homozygous for TPMT*3A. ${ }^{12}$ To date, 33 polymorphic TPMT alleles have been described, almost all of which are believed

Supplementary data for this article are available on the journal's website at www.wileyonlinelibrary.com.

'Neurology IV, Foundation IRCCS Neurological Institute "Carlo Besta," Milan, Italy

Submitted for publication 02-Nov-20I I; accepted 20-Nov-20 I I

\section{Corresponding Author:}

Pia Bernasconi, PhD, Neurology IV, Foundation Neurological Institute “Carlo Besta," Via Celoria II, 20I33 Milan, Italy.

Email: pbernasconi@istituto-besta.it 
to be associated with decreased TPMT activity. ${ }^{11,13-17}$ TPMT*2 (G238C [rs1800462]), TPMT*3A (G460A/ A719G [rs1800460/rs1142345]), TPMT*3B (G460A [rs1800460]), and TPMT*3C (A719G [rs1142345]) are the most prevalent mutant alleles, accounting for about 95\% of inherited TPMT deficiency. ${ }^{18}$ All TPMT alleles present a single mutation, except $T P M T * 3 \mathrm{~A}$ with 2 mutations, in exon 7 (G460A [rs1800460]) and exon 10 (A719G [rs1142345]), and TPMT*3D, which combines mutations encompassed within TPMT*3A with another nucleotide change in exon 5 (G292T [rs72552739]). ${ }^{14}$ These mutant alleles have been shown to encode TPMT with low or no enzyme activity, ${ }^{19}$ which undergoes rapid degradation, probably through chaperone protein-dependent, proteasome-mediated pathway and autophagy. ${ }^{16}$ In addition, intronic polymorphisms have been detected at nucleotides 114 downstream of exon 3 (T140+114A [rs3931660]), 58 downstream of exon 5 (T366+58C [rs2518463]), and 101 upstream of exon 4 (A141-101T [rs12529220]), and silent polymorphisms have been detected at nucleotides 339 in exon 5 (C339T [rs17839843]) and 474 in exon 7 (C474T [rs2842934]). ${ }^{13}$

In this study of Italian MG patients treated with AZA, we have identified a new TPMT haplotype, TPMT*3E, that was observed only in association with intolerance.

\section{Material and Methods}

Subjects. DNA samples from a cohort of 76 Italian patients affected with the autoimmune neuromuscular disease $M G$ and treated with AZA were obtained from the DNA bank of the Neurology IV Unit at Foundation Neurological Institute "Carlo Besta," Milan, Italy. Written informed consent for DNA storage and use for research purposes was obtained from all patients, as required by the ethical committee of Foundation Neurological Institute "Carlo Besta," which approved the study. All patients underwent AZA treatment and were grouped according to their response to the appropriate dose of AZA $(2-3 \mathrm{mg} / \mathrm{kg}$ per day): patients were considered responsive after at least 1 year of follow-up if they achieved pharmacological remission or had only minimal manifestations of MG according to the MGFA Post-intervention Status classification $^{20}$ or if clinical course was stable and steroid dosage had been reduced to at least $50 \%$ of pre-AZA level. Patients were classified as intolerant if they experienced persistent side effects upon AZA treatment, such as described in Ansari et al, ${ }^{21}$ which could be abrogated only by withdrawal of the drug. The remaining patients were considered nonresponders. Steroids were given in association with AZA to 66 patients $(86.8 \%)$; in the remaining 10 patients (4 intolerant, 2 unresponsive, and 4 responsive to AZA), AZA was administered alone because steroids were contraindicated or not tolerated.
Clinical data from the 76 patients, whose blood for DNA extraction had been sampled between November 2004 and July 2010, were obtained retrospectively from the records available at the Neurology IV Unit database and reviewed by a doctor blinded for the genotyping results.

The control cohort to study the frequency distribution of the newly identified variant (G420-4A) comprised 100 healthy unrelated individuals recruited randomly; of these, 83 were blood donors from the National Blood Bank of Italy (Associazione Volontari Italiani Sangue), and 17 were laboratory personnel.

DNA Extraction. Genomic DNA was extracted according to standard procedures from fresh or frozen samples of whole peripheral blood from $\mathrm{MG}$ patients and healthy individuals and stored at $-20^{\circ} \mathrm{C}$ pending assays.

$P C R$ and DNA Sequencing. The genomic structure and intron boundary sequences were derived from the NCBI's RefSeq database, access numbers NG_012137.1 (genomic sequence) and CCDS4543.1 (coding sequence). Primers specific for TPMT exons 3, 4, 6, 8, and 9 and/or intronic sequences containing known polymorphisms ${ }^{13}$ were identified using the Primer Express software package (Applied Biosystems, Foster City, California); primers specific for exons 5,7 , and 10 were according to those previously reported. ${ }^{22}$ Primer sequences and their localization are shown in Supplementary Figure S2. All primers were synthesized by NBS Biotech Service (Milan, Italy). PCR amplification was carried out in a total volume of $25 \mu \mathrm{L}$ containing $200 \mathrm{ng}$ of genomic DNA, $1 \times$ DyNAzyme Buffer (Finnzymes, Celbio, Rho, Italy), $0.2 \mathrm{mM}$ each GeneAmp dNTP (Applied Biosystems), 0.04 U DyNAzyme DNA I DNA Polymerase (Finnzymes), and $0.5 \mu \mathrm{M}$ of the specific primer pair. PCR reactions were performed on a GeneAmp PCR System 2700 (Applied Biosystems). Thermal cycling conditions to amplify exons 5,7 , and 10 were according to Schaeffeler et al. ${ }^{22}$ For all other exons, thermal cycling conditions were as follows: $94^{\circ} \mathrm{C}$ for 5 minutes, followed by 35 cycles of 30 seconds at $94^{\circ} \mathrm{C}, 30$ seconds at $55^{\circ} \mathrm{C}$, and 1 minute at $72^{\circ} \mathrm{C}$, followed by 7 minutes at $72^{\circ} \mathrm{C}$. PCR products $(10 \mu \mathrm{L})$ were confirmed on $2 \%$ ethidium bromide-stained agarose gel electrophoresis, purified ( $2.5 \mu \mathrm{L}$ of each) using ExoSAP-IT (GE Healthcare, Milan, Italy), and sequenced by NBS Biotech Service, using Big Dye terminator v3.1 Cycle Sequencing Kit (Applied Biosystems) and an ABI 3100 Genetic Analyzer (Applied Biosystems). The sequences were analyzed using SeqScape v2.1.1 (Applied Biosystems). The known polymorphisms that were analyzed in this study are listed in Supplementary Table S1.

Splice Site Prediction. Splice sites of TPMT exon 7 were predicted through BDGP: Splice Site Prediction by Neural Network (www.fruitfly.org/seq_tools/splice.html), which runs the NNSPLICE 0.9 version of the splice site predictor. 
(a)

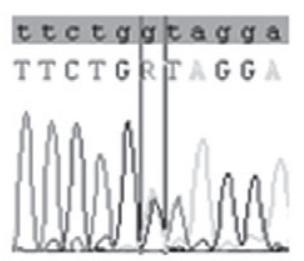

(b)

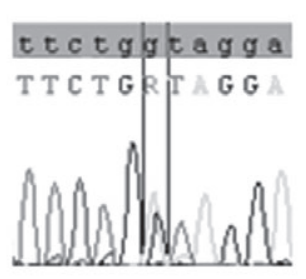

(c)

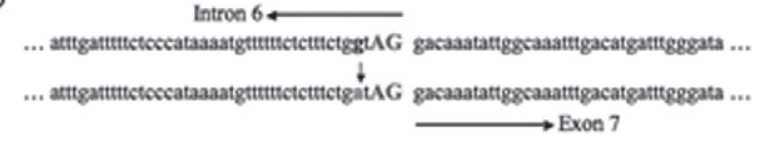

Figure I. Identification of a new TPMT polymorphism (G420-4A). Chromatograms of relevant DNA sequences in (a) MG patient and (b) healthy control; vertical lines highlight the guanine-to-adenine mutation, present in heterozygous form (R). (c) G420-4A does not affect the acceptor site for splicing of exon 6 to exon 7. Parts of the $3^{\prime}$ end of intron 6 and the $5^{\prime}$ end of exon 7 are shown for the wild-type (top) and the SNP-containing (bottom) sequences. The acceptor splice site is shown in blue; the mutated nucleotide is shown in red.

Statistical Analysis. Hardy-Weinberg equilibrium (HWE; $P>.05$ ) and minor allele frequency (MAF; $<1 \%$ ) were tested for each locus. Calculation and visualization of pairwise linkage disequilibrium (LD) between single nucleotide polymorphisms (SNPs) were carried out using the software Haploview version 4.1. Pairwise LD among the SNPs was assessed using standard coefficient $\mathrm{D}^{\prime}$ and LD coefficient expressed as $r^{2} .{ }^{23}$ We examined the degree of LD among SNPs to partition haplotype blocks using the Solid Spine algorithm in Haploview v 4.1. SNP- and haplotype-trait associations were tested within the 18-kb block region of TMPT that contains the defined SNPs.

SNP Association Analysis. Analysis was carried out using the SNPassoc R package ${ }^{24}$ through the R Foundation for Statistical Computing (Vienna, Austria; www.r-project .org). Multivariate logistic regression was used to assess the genetic effect of SNPs. Intergroup comparisons of differences in genotype frequency were performed by regression analysis for dominant, codominant, recessive, overdominant, and log-additive genetic models. Akaike's information criteria (AIC) were used to select the most parsimonious genetic model for each SNP. Odds ratios (ORs) and 95\% confidence intervals (CIs) were calculated by logistic regression analysis with adjustment for covariates of sex, age at the onset, and age at first AZA administration. To avoid false-positive results due to multiple testing, the Bonferroni correction was applied with a $P$-value cutoff of $<0.01$.

Haplotype Association Analysis. Haplotype frequencies were estimated by the expectation maximization algorithm; the inferred haplotypes with frequencies greater than 0.01 were compared between responders and intolerant patients to test associations statistically with the Haplo.score package as outlined by Schaid et al. ${ }^{25}$ The computed global score and haplotype-specific $P$ values were calculated and adjusted with clinical covariates (sex, age at the onset, age at first AZA administration) under the additive model. The $P$ values were corrected for multiple testing by 10,000-time permutation tests (Psim). The relationships between haplotype and clinical outcome were examined using a generalized linear model regression of trait-on-haplotype effect (haplo.glm function) adjusted with clinical covariates, which estimates regression coefficients corresponding to each haplotype.

\section{Results}

Patients' Responsiveness to AZA. Of the $76 \mathrm{MG}$ patients studied, 40 were classified as responsive to AZA treatment, 14 as unresponsive, and 22 as intolerant, ${ }^{21}$ with side effects including gastric intolerance (6/22 patients), hepatotoxicity ( $9 / 22$ patients), leucopenia (1/22 patients), rash (1/22 patients), neutropenia (1/22), macrocytic anemia $(1 / 22)$, and others (3/22 patients). A summary of patient data and their clinical response to AZA is shown in Supplementary Table S2.

New Intronic Polymorphism G420-4A in TPMT Gene. Because our SNP identification strategy uses primers that, in addition to the relevant exonic sequence, allow the amplification of about 50 bases of intronic sequences flanking the exon on each side (Supplementary Figure S2), we found a previously undescribed nucleotidic change (G420-4A) located 4 bases upstream of exon 7 in one intolerant patient with a heterozygous genotype (Figure 1a). This SNP was submitted (www.ncbi.nlm.nih.gov/SNP/ ftplist2.cgi?fold=ftp://ftp.ncbi.nih.gov/snp/specs/Submission/) and assigned the RefSNP ID rs56019966. We have sequenced the relevant DNA region from 100 randomly selected healthy individuals and detected this new SNP in only 1 individual (Figure 1b). Because of its position close to the beginning of exon 7 , the newly identified intronic polymorphism could affect the splicing process; however, prediction of the splice sites for exon 7 indicates that a mutation from $\mathrm{G}$ to A would not change the current acceptor site or result in a new acceptor site for splicing of exon 6 to exon 7 (Figure 1c).

Distribution of the TPMT Alleles According to AZA Responsiveness. Analysis of the distribution of TPMT alleles into the 3 different groups of patients segregated according to their responsiveness, unresponsiveness, or intolerance to AZA confirmed the presence of several known intronic and exonic TPMT polymorphisms that, singly, were not correlated with particular types of responses to AZA (Table 1), as also found in other studies. ${ }^{13,14}$ These results suggest that these polymorphisms, some of which are synonymous, do not affect the response to AZA, most likely because they do not result 
Table I. Distribution of TPMT Alleles According to AZA Responsiveness

\begin{tabular}{|c|c|c|c|c|}
\hline \multirow[b]{2}{*}{ Allele } & \multirow{2}{*}{$\begin{array}{c}\text { AZA } \\
\text { Response }\end{array}$} & \multirow{2}{*}{$\begin{array}{l}\text { Wild-Type } \\
\text { Frequency }\end{array}$} & \multicolumn{2}{|c|}{ Frequency as } \\
\hline & & & Heterozygous & Homozygous \\
\hline \multirow[t]{3}{*}{$\mathrm{A} \mid 4 \mathrm{I}-101 \mathrm{~T}$} & I & $23(52.3 \%)$ & I5 (34.1\%) & $6(13.6 \%)$ \\
\hline & $U$ & $16(57.2 \%)$ & 6 (2I.4\%) & $6(21.4 \%)$ \\
\hline & $\mathrm{R}$ & 39 (48.8\%) & 21 (26.2\%) & 20 (25\%) \\
\hline \multirow[t]{3}{*}{$\mathrm{T} 366+58 \mathrm{C}$} & 1 & 23 (52.3\%) & 15 (34.1\%) & $6(13.6 \%)$ \\
\hline & $\cup$ & I 5 (53.6\%) & 7 (25\%) & 6 (21.4\%) \\
\hline & $\mathrm{R}$ & 38 (47.5\%) & 20 (25\%) & 22 (27.5\%) \\
\hline \multirow[t]{3}{*}{ G $420-4 A^{a}$} & 1 & $43(97.7 \%)$ & I (2.3\%) & $0(0 \%)$ \\
\hline & $\cup$ & $28(100 \%)$ & $0(0 \%)$ & $0(0 \%)$ \\
\hline & $\mathrm{R}$ & $80(100 \%)$ & $0(0 \%)$ & $0(0 \%)$ \\
\hline \multirow[t]{3}{*}{$\mathrm{C} 474 \mathrm{~T}^{\mathrm{b}}$} & I & 35 (79.5\%) & 7 (15.9\%) & $2(4.6 \%)$ \\
\hline & $U$ & 20 (7I.4\%) & 4 (I4.3\%) & $4(14.3 \%)$ \\
\hline & $\mathrm{R}$ & 62 (77.5\%) & $16(20 \%)$ & 2 (2.5\%) \\
\hline \multirow[t]{3}{*}{$\mathrm{C} 339 \mathrm{~T}^{\mathrm{b}}$} & I & 44 (100\%) & $0(0 \%)$ & $0(0 \%)$ \\
\hline & U & 27 (96.4\%) & I (3.6\%) & $0(0 \%)$ \\
\hline & $\mathrm{R}$ & 79 (98.3\%) & I (I.2\%) & $0(0 \%)$ \\
\hline \multirow[t]{3}{*}{$* 3 A(* 3 B+* 3 C)$} & 1 & 44 (100\%) & $0(0 \%)$ & $0(0 \%)$ \\
\hline & $\cup$ & $27(96.4 \%)$ & I (3.6\%) & $0(0 \%)$ \\
\hline & $\mathrm{R}$ & 79 (98.3\%) & I (I.2\%) & $0(0 \%)$ \\
\hline \multirow[t]{3}{*}{$* 3 \mathrm{~A}+\mathrm{TI} 40+114 \mathrm{~A}$} & $\mathrm{I}$ & 39 (88.6\%) & 5 (I I.4\%) & $0(0 \%)$ \\
\hline & U & 28 (100\%) & $0(0 \%)$ & $0(0 \%)$ \\
\hline & $\mathrm{R}$ & $80(0 \%)$ & $0(0 \%)$ & $0(0 \%)$ \\
\hline
\end{tabular}

Abbreviations: AZA, azathioprine; I, intolerant ( $n=22$ ); $U$, unresponsive $(n=14)$; R, responsive $(n=40)$; TPMT, thiopurine S-methyltransferase. aNewly identified.

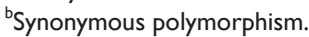

in alteration of the enzymatic function of TPMT. In contrast to what is generally accepted, we observed heterozygous $T P M T^{*} 3 \mathrm{~A}$ that was not associated with intolerance in 2 patients, 1 unresponsive and 1 responsive to AZA (Table 1); however, statistical significance could not be assessed because of the small numbers of patients.

Association Between Individual TPMT SNPs and Intolerance. As shown in Table 2, the genotype distributions of the 8 TPMT SNPs detected in our patients are all in HWE $(P>.05)$. The new SNP, G420-4A, had a minor allele frequency $(<1 \%$ of the total population; Table 2$)$ and was therefore excluded from further analysis. Logistic regression analysis revealed that under the codominant effect model assessed by AIC, T140+114A (rs3931660) was associated with intolerance after Bonferroni correction for multiple testing $(P$ value corrected $=.0039$; Table 3$)$.

Linkage Disequilibrium and Haplotype Association With Intolerance. Strong evidence for pairwise $\mathrm{LD}$ was observed between T140+114A (rs3931660) and each SNP of the *3A haplotype (G460A, rs1800460; A719G, rs1142345) with $r^{2}=.7$ (Figure 2). Using the Solid Spine method, we defined an LD block spanning $18 \mathrm{~kb}$ for the 7 relevant SNPs in the 76 patients (Figure 2). Haplotype estimation yielded 5 major haplotypes as per a frequency cutoff $>1 \%$. The test for overall distribution of haplotypes showed statistical differences in the distribution of
Table 2. Characteristics of TPMT SNPs Detected in an AZA-Treated Patient Cohort

\begin{tabular}{|c|c|c|c|c|c|}
\hline SNP & Alleles & MAF & $\begin{array}{l}\text { P-Value } \\
\text { HWE }\end{array}$ & $\begin{array}{l}\text { Gene } \\
\text { Mutation }\end{array}$ & $\begin{array}{l}\text { Amino Acid } \\
\text { Substitution }\end{array}$ \\
\hline $\begin{array}{l}\text { rs3931660 } \\
(T 140+114 A)\end{array}$ & T/A & 0.033 & 1.00 & Intronic & - \\
\hline $\begin{array}{r}\text { rs56019966 } \\
(\mathrm{G} 420-4 \mathrm{~A})\end{array}$ & $\mathrm{G} / \mathrm{A}$ & 0.007 & 1.00 & Intronic & - \\
\hline $\begin{array}{l}\text { rs } 12529220 \\
(\mathrm{~A}|4|-10 \mid \mathrm{IT})\end{array}$ & $\mathrm{A} / \mathrm{T}$ & 0.340 & 0.52 & Intronic & - \\
\hline $\begin{array}{l}\text { rsl } 17839843 \\
(\text { C339T) }\end{array}$ & $\mathrm{C} / \mathrm{T}$ & 0.013 & 1.00 & Synonymous & None \\
\hline $\begin{array}{l}\text { rs } 2518463 \\
(\mathrm{~T} 366+58 \mathrm{C})\end{array}$ & $\mathrm{T} / \mathrm{C}$ & 0.050 & 0.52 & Intronic & - \\
\hline $\begin{array}{l}\text { rs I800460 } \\
(G 460 A)\end{array}$ & $\mathrm{G} / \mathrm{A}$ & 0.046 & 1.00 & Nonsynonymous & AT \\
\hline $\begin{array}{l}\text { rs2842934 } \\
\text { (C474T) }\end{array}$ & $\mathrm{C} / \mathrm{T}$ & 0.230 & 1.00 & Synonymous & None \\
\hline $\begin{array}{l}\text { rsl| } 42345 \\
\text { (A7|9G) }\end{array}$ & $A / G$ & 0.046 & 1.00 & Nonsynonymous & YC \\
\hline
\end{tabular}

Abbreviations: AZA, azathioprine; HWE, Hardy-Weinberg equilibrium; MAF, minor allele frequency; SNP, single nucleotide polymorphisms; TPMT, thiopurine S-methyltransferase.

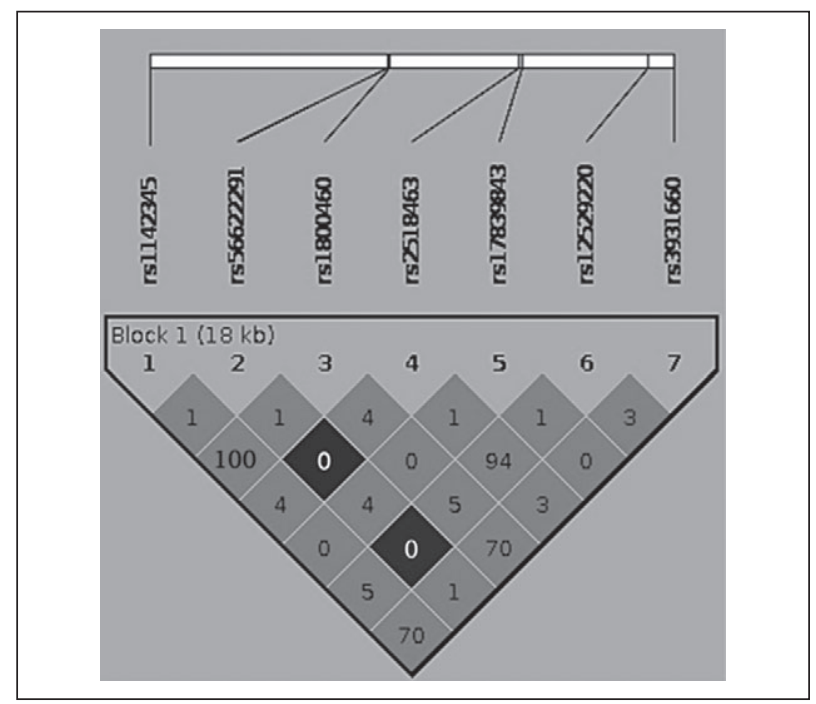

Figure 2. Haplotype-block diagram of the 18-kb TPMT region analyzed in this study. Relevant SNPs and their relative position in the TPMT gene (white bar) are indicated. Pairwise LD among the SNPs was examined using standard coefficient $D^{\prime}$ (blue: $D^{\prime}=0$; red: $D^{\prime}=1$ ) and LD coefficient expressed as $r^{2}$ (numbers in rhombi).

haplotype frequencies between intolerant and responsive patients (Table 4). Unconditional regression analysis revealed that Hap1 (ATCCACG; Table 4) was significantly associated with intolerance (adjusted OR $=2.08$ and $95 \% \mathrm{CI}=1.36-3.19$ ), compared with the most frequent haplotype Hap 3 (TACTGTA; Table 4); the simulated $P$ value was still significant after permutation 
Table 3. SNPassoc Results for Multivariate Logistic Regression Analysis With Covariate Adjustment

\begin{tabular}{|c|c|c|c|c|c|c|c|}
\hline Genetic Model & Genotype & Intolerant (\%) & Responder (\%) & OR $(95 \% \mathrm{Cl})^{\mathrm{a}}$ & $P$ Value & P-corr ${ }^{b}$ & $\mathrm{AIC}$ \\
\hline \multicolumn{8}{|l|}{ rs3931660 } \\
\hline \multirow{2}{*}{ Codominant } & $\mathrm{T} / \mathrm{T}$ & $15(75.0)$ & $33(100)$ & 1.00 (referent) & .0005 & .0039 & 66.3 \\
\hline & T/A & $5(25.0)$ & - & - & & & \\
\hline Log-additive & $0,1,2$ & $20(37.7)$ & $33(62.3)$ & - & - & - & 66.3 \\
\hline \multicolumn{8}{|l|}{ rsI2529220 } \\
\hline \multirow[t]{3}{*}{ Codominant } & $\mathrm{A} / \mathrm{A}$ & $3(15.0)$ & $5(15.2)$ & 1.00 (referent) & .73 & .11 & 79.6 \\
\hline & $\mathrm{A} / \mathrm{T}$ & $14(70.0)$ & $21(63.6)$ & $\mathrm{I} .07(0.2 \mathrm{I}-5.4 \mathrm{I})$ & & & \\
\hline & $\mathrm{T} / \mathrm{T}$ & $3(15.0)$ & 7 (2।.2) & $0.58(0.08-4.36)$ & & & \\
\hline \multirow[t]{2}{*}{ Dominant } & $\mathrm{A} / \mathrm{A}$ & $3(15.0)$ & $5(15.2)$ & $\mathrm{I} .00$ (referent) & .92 & I & 78.2 \\
\hline & $\mathrm{A} / \mathrm{T}-\mathrm{T} / \mathrm{T}$ & $17(85.0)$ & $28(84.8)$ & $0.93(0.19-4.51)$ & & & \\
\hline \multirow[t]{2}{*}{ Recessive } & $\mathrm{A} / \mathrm{A}-\mathrm{A} / \mathrm{T}$ & $17(85.0)$ & $26(78.8)$ & $\mathrm{I} .00$ (referent) & 0.43 & I & 77.6 \\
\hline & $\mathrm{T} / \mathrm{T}$ & $3(15.0)$ & $7(21.2)$ & $0.55(0.1 \mathrm{I}-2.6 \mathrm{I})$ & & & \\
\hline Overdominant & A/A-T/T & $6(30.0)$ & $12(36.4)$ & $\mathrm{I} .00$ (referent) & 0.56 & I & 77.8 \\
\hline Log-additive & $0,1,2$ & $20(37.7)$ & $33(62.3)$ & $0.75(0.28-2.02)$ & .57 & I & 77.9 \\
\hline \multicolumn{8}{|l|}{ rsl7839843 } \\
\hline \multirow[t]{2}{*}{ Codominant } & $\mathrm{C} / \mathrm{C}$ & $20(100.0)$ & $32(97.0)$ & 1.00 (referent) & 0.42 & 0.11 & 77.5 \\
\hline & $\mathrm{C} / \mathrm{T}$ & 0 & I (3.0) & & & & \\
\hline log-additive & $0,1,2$ & $20(37.7)$ & $33(62.3)$ & - & - & - & 77.5 \\
\hline \multicolumn{8}{|l|}{$r s 2518463$} \\
\hline \multirow[t]{3}{*}{ Codominant } & $\mathrm{C} / \mathrm{C}$ & $3(15.0)$ & $8(24.2)$ & 1.00 (referent) & .62 & 1 & 79.2 \\
\hline & $\mathrm{T} / \mathrm{C}$ & $14(70.0)$ & $20(60.6)$ & $2.11(0.44-9.96)$ & & & \\
\hline & $\mathrm{T} / \mathrm{T}$ & $3(15.0)$ & $5(15.2)$ & $1.90(0.26-14.08)$ & & & \\
\hline \multirow[t]{2}{*}{ Dominant } & $\mathrm{C} / \mathrm{C}$ & $3(15.0)$ & $8(24.2)$ & $\mathrm{I} .00$ (referent) & .33 & I & 77.2 \\
\hline & $\mathrm{T} / \mathrm{C}-\mathrm{T} / \mathrm{T}$ & $17(85.0)$ & $25(75.8)$ & $2.06(0.459 .44-)$ & & & \\
\hline \multirow[t]{2}{*}{ Recessive } & C/C-T/C & $17(85.0)$ & $28(84.8)$ & $\mathrm{I} .00$ (referent) & .92 & I & 78.2 \\
\hline & $\mathrm{T} / \mathrm{T}$ & $3(15.0)$ & $5(15.2)$ & I.08 (0.22-5.24) & & & \\
\hline Overdominant & $\mathrm{C} / \mathrm{C}-\mathrm{T} / \mathrm{T}$ & $6(30.0)$ & $13(39.4)$ & $\mathrm{I} .00$ (referent) & .46 & I & 77.6 \\
\hline \multicolumn{8}{|l|}{ rsl800460 } \\
\hline \multirow[t]{2}{*}{ Codominant } & G/G & $15(75.0)$ & $32(97)$ & 1.00 (referent) & 0.01 & I & 72.4 \\
\hline & $\mathrm{G} / \mathrm{A}$ & $5(25.0)$ & I (3) & II.0 (I.I2-107.5) & & & \\
\hline log-additive & $0,1,2$ & $20(37.7)$ & $33(62.3)$ & $11.0(1.12-107.5)$ & & & 72.4 \\
\hline \multicolumn{8}{|l|}{$r s 2842934$} \\
\hline \multirow[t]{3}{*}{ Codominant } & $\mathrm{C} / \mathrm{C}$ & $13(65.0)$ & $18(54.5)$ & $\mathrm{I} .00$ (referent) & .55 & I & 79.0 \\
\hline & $\mathrm{C} / \mathrm{T}$ & $6(30.0)$ & $14(42.4)$ & $0.53(0.15-1.84)$ & & & \\
\hline & $\mathrm{T} / \mathrm{T}$ & $I(5.0)$ & $I(3)$ & I.3। (0.07-26.32) & & & \\
\hline \multirow[t]{2}{*}{ Dominant } & $\mathrm{C} / \mathrm{C}$ & $13(65.0)$ & $18(54.5)$ & 1.00 & .36 & I & 77.3 \\
\hline & $\mathrm{C} / \mathrm{T}-\mathrm{T} / \mathrm{T}$ & $7(35.0)$ & $15(45.5)$ & $0.58(0.17-1.92)$ & & & \\
\hline \multirow[t]{2}{*}{ Recessive } & $\mathrm{C} / \mathrm{C}-\mathrm{C} / \mathrm{T}$ & $19(95.0)$ & $32(97)$ & 1.00 & .70 & I & 78.0 \\
\hline & $\mathrm{T} / \mathrm{T}$ & I (5.0) & I (3) & $\mathrm{I} .77(0.09-33.60)$ & & & \\
\hline Overdominant & $\mathrm{C} / \mathrm{C}-\mathrm{T} / \mathrm{T}$ & $14(70.0)$ & $19(57.6)$ & 1.00 & .28 & I & 77.0 \\
\hline & $\mathrm{C} / \mathrm{T}$ & $6(30.0)$ & $14(42.4)$ & $0.52(0.15-1.76)$ & & & \\
\hline Log-additive & $0,1,2$ & $20(37.7)$ & $33(62.3)$ & $0.70(0.24-2.0)$ & .50 & I & 77.7 \\
\hline rsII42345 & & & & & & & \\
\hline Codominant & $\mathrm{A} / \mathrm{A}$ & $15(75.0)$ & $32(97)$ & 1.00 (referent) & .01 & I & 72.4 \\
\hline & $\mathrm{A} / \mathrm{G}$ & $5(25.0)$ & I (3) & $11.0(1.12-107.5)$ & & & \\
\hline Log-additive & $0,1,2$ & $20(37.7)$ & $33(62.3)$ & II.0 (I.I2-107.5) & & & 72.4 \\
\hline
\end{tabular}

Abbreviations: AIC,Akaike's information criteria; $\mathrm{Cl}$, confidence interval; OR, odds ratio.

${ }^{a}$ Adjusted for sex, age at disease onset, and age at initiation of AZA treatment.

${ }^{\mathrm{b}} \mathrm{P}$-corr, $P$ value corrected by Bonferroni for multiple tests.

$(10,000$ times; Psim $=0.0008)$. We have designated Hap 1 as $T P M T^{*} 3 \mathrm{E}$.

Of the 5 major haplotypes estimated (Table 4), two, Hap 4 and Hap 5, had a negative Hap-Score, indicating that intolerance was unlikely in the presence of these haplotypes. In contrast, the frequency of these haplotypes was high in responders, albeit not significant. 
Table 4. Summary of Haplotypes and Their Association With Intolerance

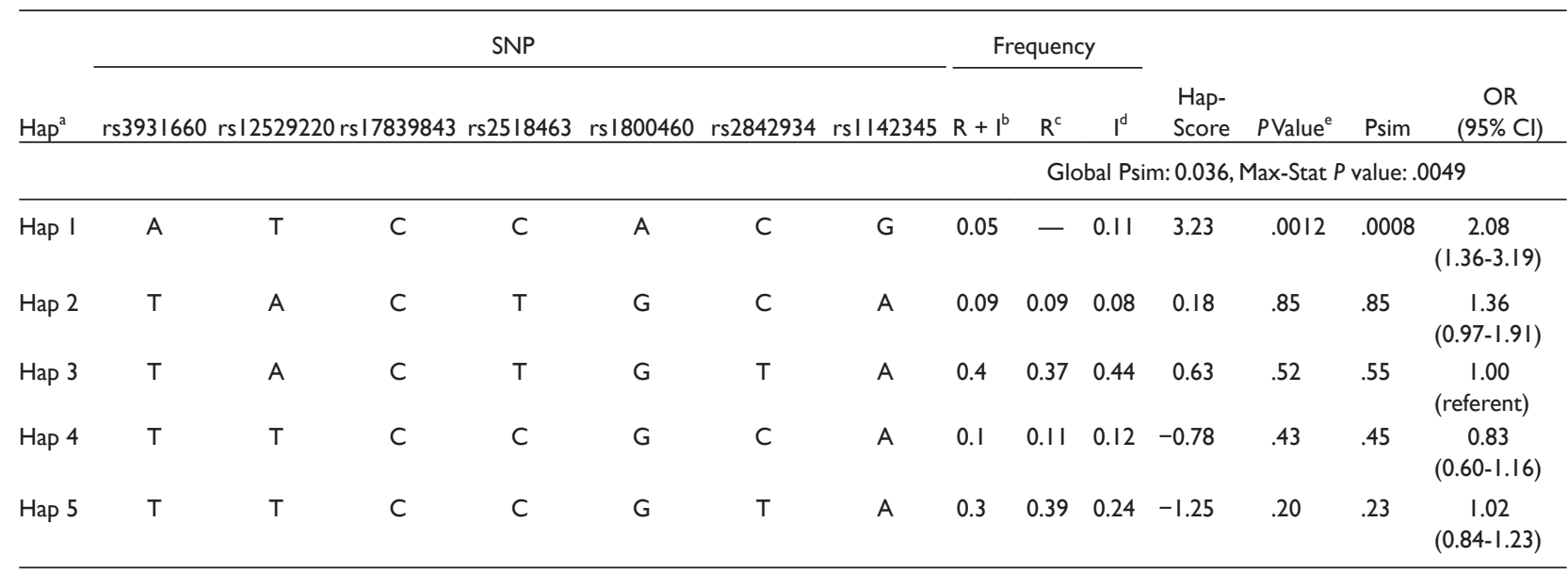

Abbreviations: $\mathrm{Cl}$, confidence interval; OR, odds ratio; SNP, single nucleotide polymorphism.

a Haplotype.

${ }^{b} \mathrm{R}+$ I Freq, haplotype frequency in intolerant and responder patient population.

${ }^{c} R$ Freq, haplotype frequency in responder patients.

I Freq, haplotype frequency in intolerant patients.

e $P$ value obtained using the haplo.score function.

\section{Discussion}

AZA is one of the most widely used immunosuppressant drugs for the treatment of autoimmune diseases such as MG, often as a steroid-sparing agent. ${ }^{1}$ However, responsiveness to the drug differs in different patients, and the possibility of predicting unresponsiveness and/or intolerance is of utmost importance for safe and effective treatment. There is increasing evidence from pharmacogenetic studies that hereditary changes in TPMT can affect the response to $\mathrm{AZA}^{18}$ by altering the functionality of this enzyme or determining its rapid degradation. ${ }^{19}$ Toward further delineation of genetic TPMT polymorphisms that might be specifically associated with the different types of response to AZA and could thereby be used as part of pharmacogenetic profiles to identify intolerant and/or unresponsive patients, we have genotyped 76 Italian MG patients treated with AZA.

We uncovered a new intronic polymorphism, G420-4A, in 1 of the 22 intolerant patients, as well as in 1 of the 100 healthy controls investigated. As this polymorphism is located 4 bases upstream of the RNA splicing site for exon 7, we speculated that it could affect mRNA splicing, ${ }^{26,27}$ thus coding for a protein with defective enzymatic activity. However, splice site prediction indicated that the mutation does not affect the relevant acceptor site, nor does it create a new splicing site. Nevertheless, we cannot exclude that this SNP could interfere with the binding of a relevant protein of the spliceosome, thereby affecting mRNA splicing and the protein product. Since the variant G420-4A was found in only 1 patient and 1 control individual, further studies in larger cohorts of varying ethnicities will be necessary to better understand if this SNP has functional implication on TPMT enzyme activity and to evaluate its frequency in the general population.

The TPMT*3A haplotype has been linked to intolerance to $\mathrm{AZA}^{18}$ and has not so far been reported in association with responsiveness or unresponsiveness. In our study, however, the TPMT*3A haplotype was detected in 1 unresponsive patient and in 1 apparently responsive patient by DNA sequencing (Table 1) that was confirmed by allelic discrimination analysis (data not shown). This responsive $T P M T^{*} 3 \mathrm{~A}$ patient was given AZA as a steroidsparing agent. Whether or not steroid-sparing in this $T P M T * 3$ A patient was directly correlated to AZA treatment is not absolutely established. Nevertheless, after 11 years of AZA treatment, the patient's daily steroid dose had been reduced more than $80 \%$ from the initial dose. Neither this patient nor the unresponsive one experienced side effects as might be expected in individuals carrying the TPMT*3A allele. ${ }^{14}$ The unexpected occurrence of $T P M T^{*} 3 \mathrm{~A}$ in association with unresponsiveness or responsiveness in our cohort could be related to 1 or more additional unknown variants in TPMT or in other genes of AZA metabolism that would abrogate the effect of the TPMT*3A allele, and although such an effect has never been demonstrated, it could lead to hyperactivity of the enzyme in the unresponsive patient. This could occur also at the genomic level via epistasis by modifier genes. ${ }^{28,29}$ Another possibility would involve a variation in $G S T$ resulting in reduced enzymatic activity and thereby only little conversion of AZA to 6-MP, hence unresponsiveness, irrespective of the TPMT*3A mutations that affect TPMT downstream of GST in the pathway. Finally, unresponsiveness could be accounted for by variation(s) in $A O X 1$ coding for aldehyde oxidase, which contributes to the catabolism of AZA, MP, and other thiopurine 
metabolites. Indeed, the presence of a coding-region SNP in $A O X 1$ is significantly associated with lack of therapeutic response to AZA, which in this case is apparently not related to differences in TGN levels. ${ }^{30}$ The TPMT*3A haplotype appears to be the most frequent inactivating mutant allele in Caucasians $(3.2 \%-5.7 \%),{ }^{31-33}$ including Italian subjects (3.9\%). ${ }^{34}$ In our cohort, the percentage of patients heterozygous for $T P M T^{*} 3 \mathrm{~A}$ mutations was more than twice as high (7/76 patients; $9.2 \%)$, a difference that could be related to the small cohort studied.

Through genotyping of the TMPT gene, we detected a novel haplotype defined by 7 SNPs, which we have termed $T P M T * 3 \mathrm{E}$. TPMT*3E was significantly associated with intolerance in our population after covariate adjustment (Table 4; Psim $=0.0026$ ), and none of the 14 unresponsive or 40 responsive patients carried this novel haplotype. The T140+114A polymorphism (rs3931660) was found in our cohort only in association with the TPMT*3A mutations, as part of TPMT*3E (5/22 intolerant patients); none of the patients carried this SNP on its own. At present, it is not possible to ascertain if the presence of the T140+114A polymorphism amplifies the intolerant response to the drug that is associated with mutations encompassed in TPMT*3A. Studies on larger cohorts will be needed to determine whether this SNP is always found as part of the TPMT*3E haplotype or also occurs alone, and if so, whether it could by itself be associated with intolerance. In this context, the study of Otterness et al, ${ }^{13}$ who first reported this SNP, is not clear. Larger, diverse cohorts are also necessary to investigate ethnic variations in the frequency of $T P M T^{*} 3 \mathrm{E}$ or the single T140+114A polymorphism. Indeed, the frequency of TPMT alleles in different ethnic groups varies greatly; for example, $T P M T^{*} 3 \mathrm{~A}$, which is the most prominent allele in Caucasians, was not found in Ghanaian and Korean individuals, ${ }^{32,35,36}$ and the frequency of TPMT*3C, the most prominent allele in Ghanaian and Korean individuals, is very low in Caucasians. ${ }^{30} \mathrm{We}$ could find reports only for T140+114A frequencies for 2 different populations in the NCBI SNP database (www.ncbi.nlm.nih.gov/ projects/SNP/snp_ref.cgi?rs $=3931660$ ), one of $6 \%$ for a Nigerian cohort of 25 individuals and one of $9.4 \%$ for a cohort of 90 individuals whose ethnic origin is not reported. It is therefore difficult to discuss if the allele frequency for T140+114A in our AZA-treated Italian cohort (3.2\%) is commensurate with that of other populations.

Strikingly, whereas the TPMT*3A haplotype was seen also in the context of unresponsiveness or responsiveness in our cohort, none of the unresponsive or responsive patients carried the TPMT*3E haplotype. This could suggest a very strong link of $T P M T * 3 \mathrm{E}$ with AZA intolerance.
While the association of the new TPMT*3E haplotype with intolerance to AZA must be further delineated, these studies confirm the relevance of establishing pharmacogenetic profiles for specific drugs, especially those that can result in severe side effects, to determine the suitability of AZA treatment in patients. Thus, pharmacogenetic profiling for AZA should not only include studies on TPMT but also incorporate studies on all the other genes of the AZA metabolic pathways that could play a role in the response to AZA, in particular the genes coding for GST and xanthine oxidase or aldehyde oxidase, which, respectively, convert AZA into 6-MP and detoxify the drug. ${ }^{30,37,38}$ Indeed, these, which could be key enzymes in determining responsiveness, unresponsiveness, or intolerance, represent future targets for our pharmacogenetics studies of the response to AZA. In this context, a significant association has been demonstrated for an aldehyde oxidase gene variant and lack of therapeutic response to $\mathrm{AZA}^{30}{ }^{30}$ whereas intolerance to the drug has also been linked to polymorphisms in multidrug-resistance protein 4 and ecto-5'-nucleotidase. ${ }^{39,40}$

\section{Declaration of Conflicting Interests}

The author(s) declared no potential conflicts of interest with respect to the research, authorship, and/or publication of this article.

\section{Funding}

This work was supported by the Italian Ministry of Health 2009-2010 (Annual Research Funding RC2009/LR5 and RC2010/R5), Associazione Italiana Miastenia e Malattie Immunodegenerative Amici del Besta-Onlus (AIM), and the European Commission (FP7 HEALTH-2009-242-210).

\section{References}

1. Richman D, Agius M. Treatment of autoimmune myasthenia gravis. Neurology. 2003;61(12):1652-1661.

2. Mantegazza R, Antozzi C, Peluchetti D, Sghirlanzoni A, Cornelio F. Azathioprine as a single drug or in combination with steroids in the treatment of myasthenia gravis. J Neurol. 1988;235(8): 449-453.

3. Cendrowski W. Therapeutic trial of Imuran (azathioprine) in multiple sclerosis. Acta Neurol Scand. 1971;47(2):254-260.

4. Suarez-Almazor M, Spooner C, Belseck E. Azathioprine for treating rheumatoid arthritis. Cochrane Database Syst Rev. 2000 (4):CD001461.

5. Lee A, Farrell G. Mechanism of azathioprine-induced injury to hepatocytes: roles of glutathione depletion and mitochondrial injury. J Hepatol. 2001;35(6):756-764.

6. Coulthard S, Hogarth L, Little M, et al. The effect of thiopurine methyltransferase expression on sensitivity to thiopurine drugs. Mol Pharmacol. 2002;62(1):102-109.

7. Lennard L, Lilleyman J, Van Loon J, Weinshilboum R. Genetic variation in response to 6-mercaptopurine for childhood acute lymphoblastic leukaemia. Lancet. 1990;336(8709):225-229. 
8. Wang L, Weinshilboum R. Thiopurine S-methyltransferase pharmacogenetics: insights, challenges and future directions. Oncogene. 2006;25(11):1629-1638.

9. de Boer N, Mulder C, van Bodegraven A. Myelotoxicity and hepatotoxicity during azathioprine therapy. Neth JMed. 2005;63(11):444-446.

10. Lennard L, Van Loon J, Weinshilboum R. Pharmacogenetics of acute azathioprine toxicity: relationship to thiopurine methyltransferase genetic polymorphism. Clin Pharmacol Ther. 1989;46(2):149-154.

11. Krynetski E, Schuetz J, Galpin A, et al. A single point mutation leading to loss of catalytic activity in human thiopurine S-methyltransferase. Proc Natl Acad Sci U S A. 1995;92(4):949-953.

12. Weinshilboum R, Sladek S. Mercaptopurine pharmacogenetics: monogenic inheritance of erythrocyte thiopurine methyltransferase activity. Am J Hum Genet. 1980;32(5):651-662.

13. Otterness D, Szumlanski C, Lennard L, et al. Human thiopurine methyltransferase pharmacogenetics: gene sequence polymorphisms. Clin Pharmacol Ther. 1997;62(1):60-73.

14. Garat A, Cauffiez C, Renault N, et al. Characterisation of novel defective thiopurine S-methyltransferase allelic variants. Biochem Pharmacol. 2008;76(3):404-415.

15. Kham SK, Soh CK, Aw DC, Yeoh AE. TPMT*26 $(208 \mathrm{~F} \rightarrow \mathrm{L})$, a novel mutation detected in a Chinese. Br J Clin Pharmacol. 2009;68(1):120-123.

16. Feng Q, Vannaprasaht S, Peng Y, et al. Thiopurine S-methyltransferase pharmacogenetics: functional characterization of a novel rapidly degraded variant allozyme. Biochem Pharmacol. 2010;79(7):1053-1061.

17. Appell ML, Wennerstrand $\mathrm{P}$, Peterson C, Hertervig E, Mårtensson LG. Characterization of a novel sequence variant, TPMT*28, in the human thiopurine methyltransferase gene. Pharmacogenet Genomics. 2010;20(11):700-707.

18. Weinshilboum R. Pharmacogenomics: catechol O-methyltransferase to thiopurine S-methyltransferase. Cell Mol Neurobiol. 2006;26(4-6):539-561.

19. Ujiie S, Sasaki T, Mizugaki M, Ishikawa M, Hiratsuka M. Functional characterization of 23 allelic variants of thiopurine S-methyltransferase gene (TPMT*2-*24). Pharmacogenet Genomics. 2008;18(10):887-893

20. Jaretzki AR, Barohn R, Ernstoff R, et al. Myasthenia gravis: recommendations for clinical research standards. Task Force of the Medical Scientific Advisory Board of the Myasthenia Gravis Foundation of America. Neurology. 2000;55(1):16-23.

21. Ansari A, Arenas M, Greenfield S, et al. Prospective evaluation of the pharmacogenetics of azathioprine in the treatment of inflammatory bowel disease. Aliment Pharmacol Ther. 2008;28(8):973-983.

22. Schaeffeler E, Lang T, Zanger U, Eichelbaum M, Schwab M. Highthroughput genotyping of thiopurine S-methyltransferase by denaturing HPLC. Clin Chem. 2001;47(3):548-555.

23. Barrett JC, Fry B, Maller J, Daly MJ. Haploview: analysis and visualization of $\mathrm{LD}$ and haplotype maps. Bioinformatics. 2005;21(2):263-265.

24. González JR, Armengol L, Solé X, et al. SNPassoc: an R package to perform whole genome association studies. Bioinformatics. 2007;23(5):644-645.
25. Schaid DJ, Rowland CM, Tines DE, Jacobson RM, Poland GA. Score tests for association between traits and haplotypes when linkage phase is ambiguous. Am J Hum Genet. 2002;70(2):425-434.

26. Palaniswamy R, Teglund S, Lauth M, Zaphiropoulos PG, Shimokawa T. Genetic variations regulate alternative splicing in the $5^{\prime}$ untranslated regions of the mouse glioma-associated oncogene 1, Gli1. BMC Mol Biol. 2010;11:32.

27. Hull J, Campino S, Rowlands K, et al. Identification of common genetic variation that modulates alternative splicing. PLoS Genet. 2007;3(6):e99

28. Rochette J, Le Gac G, Lassoued K, Férec C, Robson K. Factors influencing disease phenotype and penetrance in HFE haemochromatosis. Hum Genet. 2010;128(3):233-248.

29. Meisler M, O'Brien J, Sharkey L. Sodium channel gene family: epilepsy mutations, gene interactions and modifier effects. J Physiol. 2010;588(pt 11):1841-1848.

30. Smith M, Marinaki A, Arenas M, et al. Novel pharmacogenetic markers for treatment outcome in azathioprine-treated inflammatory bowel disease. Aliment Pharmacol Ther. 2009;30(4):375-384.

31. Spire-Vayron de la Moureyre C, Debuysere H, Mastain B, et al. Genotypic and phenotypic analysis of the polymorphic thiopurine S-methyltransferase gene (TPMT) in a European population. $\mathrm{Br} J$ Pharmacol. 1998;125(4):879-887.

32. Ameyaw M, Collie-Duguid E, Powrie R, Ofori-Adjei D, McLeod H. Thiopurine methyltransferase alleles in British and Ghanaian populations. Hum Mol Genet. 1999;8(2):367-370.

33. McLeod H, Pritchard S, Githang'a J, et al. Ethnic differences in thiopurine methyltransferase pharmacogenetics: evidence for allele specificity in Caucasian and Kenyan individuals. Pharmacogenetics. 1999;9(6):773-776.

34. Rossi A, Bianchi M, Guarnieri C, Barale R, Pacifici G. Genotypephenotype correlation for thiopurine S-methyltransferase in healthy Italian subjects. Eur J Clin Pharmacol. 2001;57(1):51-54.

35. Schaeffeler E, Zanger U, Eichelbaum M, et al. Highly multiplexed genotyping of thiopurine s-methyltransferase variants using MALD-TOF mass spectrometry: reliable genotyping in different ethnic groups. Clin Chem. 2008;54(10):1637-1647.

36. Cheon J, Kim J, Kim B, et al. Allele frequency of thiopurine methyltransferase and inosine triphosphate pyrophosphatase gene polymorphisms in Korean patients with inflammatory bowel diseases. Hepatogastroenterology. 2009;56(90):421-423.

37. Lennard L, Maddocks J. Assay of 6-thioguanine nucleotide, a major metabolite of azathioprine, 6-mercaptopurine and 6-thioguanine, in human red blood cells. J Pharm Pharmacol. 1983;35(1):15-18.

38. Cara C, Pena A, Sans M, et al. Reviewing the mechanism of action of thiopurine drugs: towards a new paradigm in clinical practice. Med Sci Monit. 2004;10(11):RA247-RA254.

39. Li F, Fridley B, Matimba A, et al. Ecto-5'-nucleotidase (NT5E) and thiopurine cellular circulation: association with cytotoxicity. Drug Metab Dispos. 2010;38(12):2329-2338.

40. Ban $H$, Andoh A, Imaeda $H$, et al. The multidrug-resistance protein 4 polymorphism is a new factor accounting for thiopurine sensitivity in Japanese patients with inflammatory bowel disease. J Gastroenterol. 2010;45(10):1014-1021. 